\title{
A new perspective on correlated polyelectrolyte adsorption: Positioning, conformation, and patterns
}

Sandra C. C. Nunes, Tânia F. G. G. Cova, and A. A. C. C. Pais

Citation: The Journal of Chemical Physics 139, 054906 (2013); doi: 10.1063/1.4817338

View online: https://doi.org/10.1063/1.4817338

View Table of Contents: http://aip.scitation.org/toc/jcp/139/5

Published by the American Institute of Physics

\section{Articles you may be interested in}

Electrostatic correlations and the polyelectrolyte self energy

The Journal of Chemical Physics 146, 084901 (2017); 10.1063/1.4975777

Pair interactions in polyelectrolyte-nanoparticle systems: Influence of dielectric inhomogeneities and the partial dissociation of polymers and nanoparticles

The Journal of Chemical Physics 143, 164904 (2015); 10.1063/1.4934242

Electrostatic origins of polyelectrolyte adsorption: Theory and Monte Carlo simulations

The Journal of Chemical Physics 133, 044906 (2010); 10.1063/1.3463426

Perspective: Outstanding theoretical questions in polymer-nanoparticle hybrids

The Journal of Chemical Physics 147, 020901 (2017); 10.1063/1.4990501

Single chains of strong polyelectrolytes in aqueous solutions at extreme dilution: Conformation and counterion distribution

The Journal of Chemical Physics 145, 144903 (2016); 10.1063/1.4964649

Adsorption of polymer chains at surfaces: Scaling and Monte Carlo analyses

The Journal of Chemical Physics 77, 6296 (1982); 10.1063/1.443835

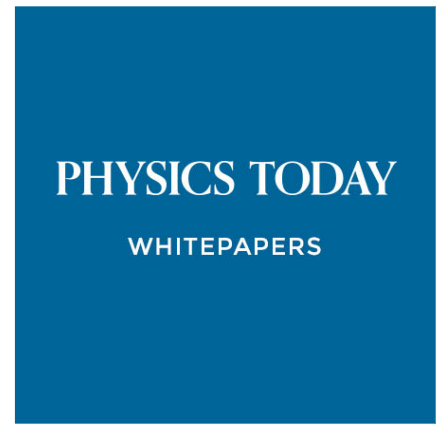

ADVANCED LIGHT CURE ADHESIVES

Take a closer look at what these environmentally friendly adhesive systems can do

\section{READ NOW}

PRESENTED BY 8) MASTERBOND 


\title{
A new perspective on correlated polyelectrolyte adsorption: Positioning, conformation, and patterns
}

\author{
Sandra C. C. Nunes, ${ }^{a}$ Tânia F. G. G. Cova, and A. A. C. C. Pais \\ Chemistry Department, University of Coimbra, Rua Larga, 3004-535 Coimbra, Portugal
}

(Received 5 June 2013; accepted 19 July 2013; published online 7 August 2013)

\begin{abstract}
This work focuses on multiple chain deposition, using a coarse-grained model. The phenomenon is assessed from a novel perspective which emphasizes the conformation and relative arrangement of the deposited chains. Variations in chain number and length are considered, and the surface charge in the different systems ranges from partially neutralized to reversed by backbone deposition. New tools are proposed for the analysis of these systems, in which focus is given to configuration-wise approaches that allow the interpretation of correlated multi-chain behavior. It is seen that adsorption occurs, with a minimal effect upon the bulk conformation, even when overcharging occurs. Also, chain ends create a lower electrostatic potential, which makes them both the least adsorbed region of the backbone, and the prevalent site of closer proximity with other chains. Additionally, adsorption into the most favorable region of the surface overrides, to a large degree, interchain repulsion.
\end{abstract} (C) 2013 AIP Publishing LLC. [http://dx.doi.org/10.1063/1.4817338]

\section{INTRODUCTION}

The interaction between polyelectrolytes and surfaces plays an important role in a large number of applications including coating processes, ${ }^{1,2}$ gene and drug delivery, ${ }^{3,4}$ and sensor development ${ }^{5-7}$ among several others. ${ }^{8-12}$ Tailoring surface properties permit to optimize a specific application ${ }^{4,13-17}$ and a deep understanding of the adsorption process is fundamental to guide experimental design.

Several studies have addressed the adsorption of polyelectrolytes onto surfaces ${ }^{18-26}$ and, particularly, the adsorption of multiple chains has been largely explored, ${ }^{17,22,27-30}$ considering either the deposition of mixtures of oppositely charged polyelectrolytes ${ }^{17,22,30,31}$ or focusing only onto cationic $^{1,23,32,33}$ or anionic polyelectrolytes. ${ }^{24,34}$ Some situations in which irregularly distributed or mobile charges are present have been previously addressed, ${ }^{35,36}$ showing that a number of effects are present including surfacepolyelectrolyte charge correlation. More recently, a growing interest in anionic amphiphilic polyelectrolytes, has emerged, particularly motivated by their potential in the biomedical fields. ${ }^{37-39}$

In spite of the amount of work and variety of aspects covered, the study of the spatial distribution of the polyelectrolytes in the surface is still a largely unexplored subject. The main focus of the present work is the characterization of the coverage of the surface, with identification of the differentially populated regions, and establishing the distance between polyelectrolyte chains and the profile of vicinity of the backbones. Controlling the surface properties and, in particular knowing the arrangement of the adsorbed polyelectrolytes is crucial in a vast number of fields involving polyelectrolyte deposition, as the properties of the final surface are almost entirely controlled by the characteristics of the

\footnotetext{
a)Electronic mail: snunes@qui.uc.pt
}

deposited layer. For example, in procedures involving selfassembly, it is expected that the homogeneity of the polyelectrolyte layer formed and the conformation of the adsorbed chains might affect the properties of the coating and thus constrain the subsequent interaction with further molecules. The conformation of the adsorbed backbones also impacts on the layer thickness, which is another important issue in many applications.

To address the proposed goals, the approach developed in a previous study ${ }^{40}$ was extended to the problem of likecharged multichain adsorption onto regularly charged surface patches. Focus is given to the ability of the surface to accommodate an increasing number of polyelectrolyte chains of different length, and particular attention is devoted to the arrangement of these chains on the surface upon adsorption. Aspects such as chain conformation, multiple chain adsorption pattern, and the factors behind the organization of the backbones on the surface are explored in detail using Monte Carlo simulations in a coarse-grained model. Two sets of systems are considered. In the first one, a polyelectrolyte chain was sequentially fragmented into an increasing number of shorter chains. This imposes, in all systems, the same number (concentration) of monomers interacting with the surface and allows assessing the importance of molecular weight. In the second case, the polyelectrolyte chain length was kept constant, and an increasing number of chains were considered, promoting an increasing monomer concentration and surface coverage. In both cases, the conformation of the chains, the respective distribution on the surface, and the relative positioning was inspected.

Results indicate that adsorption occurs mainly without a significant compaction of the polyelectrolyte chains in relation to the bulk conformation. This is valid even for systems in which surface overcharging is present. Furthermore, the arrangement of the chains upon adsorption seems to be mainly governed by a balance between chain interaction and deposition in regions where surface attraction is favored. 


\section{METHODOLOGY}

\section{A. Simulation details}

A simple model was adopted to describe the interaction between negatively charged polyelectrolyte chains in aqueous solution and a finite positively charged surface. In the model, the polyelectrolyte is represented by a spring-bead chain described as a sequence of negatively charged hard spheres connected with harmonic bonds, being the chain flexibility regulated by angular force terms. The charged surface is taken to be a hard planar wall with embedded positively charged fixed hard spheres positioned in the $x y$ plane in a regular fashion. The spacing between the charges is constant and imposes the surface charge density. Monovalent simple ions, also treated as charged hard spheres, corresponding to the counterions of both the surface and the polyelectrolyte chains are explicitly included. The solvent enters the system only through its relative permittivity.

A box with dimensions $L_{x}=L_{y}=2000 \AA$ and hard walls placed at $z= \pm 1000 \AA$ was imposed. The size of the simulation box $(2000 \times 2000 \times 2000 \AA)$ is large enough so that boundary effects are negligible.

The system is periodic in the $x$ and $y$ directions. The surface, formed by the charged hard-spheres is positioned at the center of the simulation box in the $x y$ plane and is considerably smaller $(160 \times 160 \AA)$ than the corresponding box area. Thus, surface charges are distributed in a square grid from $-80 \AA$ to $+80 \AA$ in both the $x$ and $y$ directions, while the simulation box ranges from $-1000 \AA$ to $+1000 \AA$ along these axes. A hard-sphere radius of $2.0 \AA$ was used for all the species. The temperature $\mathrm{T}=298.15 \mathrm{~K}$ and relative permittivity $\epsilon_{r}=78.4$ were considered throughout. In most experimental conditions, the dielectric constants of the solvent and the surface are different, and the presence of the polyion near the surface induces a polarization of both mediums. This effect has been accounted for in some works by considering the presence of image charges. ${ }^{21,33,41,42}$ It will not be, however, focused in the present work.

All interactions were taken as pairwise additive. The total potential energy, $U$, of the system can be expressed as a sum of four contributions: the nonbonded potential energy, $U_{\text {nonbond }}$, the bond potential energy, $U_{\text {bond }}$, the angular potential energy, $U_{\text {ang }}$, and the confining external potential energy, $U_{\text {ext }}$. The nonbonded potential energy is given by

$$
U_{\text {nonbond }}=\sum_{i<j} u_{i, j}\left(r_{i, j}\right),
$$

where the summation extends over chain beads, surface charges, and counterions, with $u_{i, j}$ representing the electrostatic potential plus a hard-sphere repulsion according to

$$
u_{i, j}\left(r_{i, j}\right)=\left\{\begin{array}{cc}
\infty & r_{i, j}<R_{i}+R_{j} \\
\frac{Z_{i} Z_{j} e^{2}}{4 \pi \epsilon_{0} \epsilon_{r}} \frac{1}{r_{i, j}} & r_{i, j} \geq R_{i}+R_{j}
\end{array},\right.
$$

where $Z_{i}$ is the valence of the particle $i, R_{i}$ is the radius of particle $i, r_{i, j}$ is the distance between particles $i$ and $j, e$ is the elementary charge, $\epsilon_{o}$ is the permittivity of vacuum, and $\epsilon_{r}$ is the relative permittivity of the solvent. Polyelectrolyte chain beads are connected by harmonic bonds, and the bond potential energy of the polyelectrolyte is given by

$$
U_{\text {bond }}=\sum_{c=1}^{N_{c}} \sum_{i=1}^{N_{\text {bead }, c}-1} \frac{k_{\text {bond }}}{2}\left(r_{i, i+1}-r_{0}\right)^{2},
$$

where $N_{c}$ is the number of chains and $N_{b e a d, c}$ is the number of beads of chain c, $r_{i, i+1}$ is the distance between two connected beads with the equilibrium separation $r_{0}=5.0 \AA$, and the force constant $k_{\text {bond }}=0.4 \mathrm{~N} \mathrm{~m}^{-1}$. With the other interactions included, the typical root-mean-square (rms) bead-bead separation becomes $\left\langle R_{\text {bead, bead }}^{2}\right\rangle^{1 / 2} \approx 5.8 \AA$. The angular potential energy, $U_{a n g}$, is given by

$$
U_{\text {ang }}=\sum_{c=1}^{N_{c}} \sum_{i=1}^{N_{\text {bead }, c}-1} \frac{k_{\text {ang }}}{2}\left(\alpha_{i, i+1}-\alpha_{0}\right)^{2},
$$

where $\alpha_{i}$ is the angle formed by the vectors $r_{i+1}-r_{i}$ and $r_{i-1}-r_{i}$ with the equilibrium angle $\alpha_{0}=180^{\circ}$ and the force constant $k_{\text {ang }}$. The value $k_{\text {ang }}=3.44 \times 10^{-24} \mathrm{~J} \mathrm{deg}^{-2}$ was used throughout the work. Finally, the confining external potential energy, $U_{\text {ext }}$, is given by

$$
U_{e x t}=\sum_{i} u_{e x t}\left(z_{i}\right)
$$

where the summation extends only over polyelectrolyte beads and counterions with

$$
u_{\text {ext }}\left(z_{i}\right)=\left\{\begin{array}{cc}
\infty & \left|z_{i}\right|>z_{\text {wall }} \\
0 & \left|z_{i}\right|<z_{\text {wall }}
\end{array} .\right.
$$

All Monte Carlo simulations were performed with the MOLSIM $^{43}$ simulation package in the canonical ensemble and the Metropolis algorithm. ${ }^{44}$ Three types of Monte Carlo trial moves were employed for the polyelectrolyte chain: single bead move, translation of the entire chain, and slithering move where one of the end beads is moved to the opposite end of the chain. The single particle move was attempted 100 times more often than the other two types of moves. The chain and surface counterions were subjected to translational moves and the surface charges were fixed. Each simulation included an equilibration of at least $5 \times 10^{6}$ trial moves per particle followed by a production run of at least the same number of moves which was found to be sufficient to warrant convergence.

The influence of the interaction between polyelectrolytes and surface is inspected considering a fixed and uniform surface charge density with the 100 surface charges that constitute the adsorbing surface regularly distributed with a closest distance between charges of $16 \AA$. With this arrangement, the mean charge density of the surface is $0.063 \mathrm{C} \mathrm{m}^{-2}$ which is within the values found in the literature (0.0016$\left.0.368 \mathrm{C} \mathrm{m}^{-2}\right) .^{23,27,30,45-47}$

\section{B. Properties}

\section{Conformation and adsorption}

The variables considered in the simulation are the number and length of the polyelectrolyte chains. Adsorption was evaluated resorting to the properties describing the spatial extension of the chains and their conformation with respect to 
the surface. Chain monomers located within $8 \AA$ from the plane of surface charges were considered to be adsorbed. The extension of the polyelectrolyte backbones was characterized by the radius of gyration $\left(R_{g}\right)$ and by the respective projections parallel $\left(R g_{x y}\right)$ and perpendicular to the surface $\left(R g_{z}\right)$ according to

$$
\begin{gathered}
\left\langle R_{\mathrm{g}}^{2}\right\rangle^{1 / 2}=\left\langle\frac{1}{N_{\mathrm{mon}}} \sum_{i=1}^{N_{\text {mon }}}\left(r_{i}-r_{\mathrm{CM}}\right)^{2}\right\rangle^{1 / 2} \\
\left\langle R_{\mathrm{g}_{\mathrm{xy}}}^{2}\right\rangle^{1 / 2}=\left\langle\frac{1}{N_{\text {mon }}} \sum_{i=1}^{N_{\text {mon }}}\left[\left(x_{i}-x_{\mathrm{CM}}\right)^{2}+\left(y_{i}-y_{\mathrm{CM}}\right)^{2}\right]\right\rangle^{1 / 2} \\
\left\langle R_{\mathrm{g}_{\mathrm{z}}}^{2}\right\rangle^{1 / 2}=\left\langle\frac{1}{N_{\mathrm{mon}}} \sum_{i=1}^{N_{\text {mon }}}\left(z_{i}-z_{\mathrm{CM}}\right)^{2}\right\rangle^{1 / 2}
\end{gathered}
$$

where $r$ denotes the position and $x, y$, and $z$ the coordinates. The subindexes $i$ and CM refer to each $i$ segment and to the center of mass of the chain, respectively.

\section{Spatial distribution and proximity analysis}

The spatial distribution of the chains in the surfaces was inspected resorting to positioning density maps obtained using Gnuplot v4.4, and also to metric approaches based on proximity profiles. These evaluate the distribution profile of the distances between the central monomers of the chains, and between selected monomers.

The algorithms used for proximity analysis were implemented and optimized by the authors using Octave v3.2.4. ${ }^{48}$ The analysis is based on three procedures. The first relies on the determination of the nearest-neighbor distances between chains (represented by their central monomer) in a minimum of 400 configurations obtained at regular intervals from the total number of steps in the production run. These distances are collected in a relative frequency plot. The second uses the nearest-neighbor distance of each monomer relative to the monomers of the other chains, which are averaged for all chains and configurations. From these averaged values, the profile of closest distance for the monomers is established. Finally, the index of the nearest-neighbor in other chain is identified for each end monomer. The relative frequency of indexes value is subsequently compiled. The same procedure is applied for the central monomer, represented by that of lower rank and closer to the center of the chain. In summary, the first procedure establishes a distribution of closest distance between chains. The second one establishes a profile of proximity to each monomer. The last one allows to readily inspect which monomers are more frequently closer to the chain ends, or to the center. The combination of these procedures provides a detailed description of the arrangement of the chains relatively to each other.
TABLE I. Overview of the systems under study, with indication of the number of chains, $N_{\text {chains }}$, number of monomers in each chain, $N_{\text {mon/chain }}$, ratio between the total number of charges in chains and the number of surface charges, $Q_{\text {chains }} / Q_{\text {surf }}$, chain length, $L_{\text {chain }}$, (obtained in the bulk) and label C $x$ n $y$, where $x$ denotes the number of chains in the system and $y$ the number of monomers in each chain.

\begin{tabular}{lcccc}
\hline \hline$N_{\text {chains }}$ & $N_{\text {mon/chain }}$ & $Q_{\text {chains }} / Q_{\text {surf }}$ & $L_{\text {chain }}(\AA)$ & Label \\
\hline Set 1 & & & & \\
1 & 60 & 0.6 & 271.7 & C1n60 \\
2 & 30 & 0.6 & 131.1 & C2n30 \\
3 & 20 & 0.6 & 85.3 & C3n20 \\
4 & 15 & 0.6 & 62.8 & C4n15 \\
5 & 12 & 0.6 & 49.7 & C5n12 \\
6 & 10 & 0.6 & 41.2 & C6n10 \\
Set 2 & 20 & & & \\
1 & 20 & 0.2 & 85.3 & C1n20 \\
2 & 20 & 0.4 & 85.3 & C2n20 \\
3 & 20 & 0.6 & 85.3 & C3n20 \\
4 & 20 & 0.8 & 85.3 & C4n20 \\
5 & 20 & 1.0 & 85.3 & C5n20 \\
6 & 20 & 1.2 & 85.3 & C6n20 \\
7 & & 1.4 & 85.3 & C7n20 \\
\hline \hline
\end{tabular}

\section{RESULTS AND DISCUSSION}

Monte Carlo simulations were performed in order to explore the conformation and spatial distribution of an increasing number of polyelectrolyte chains adsorbing onto a regularly oppositely charged finite surface. Two different cases were considered. In one, a chain was sequentially fragmented into an increasing number of consecutively smaller chains. In the other, the chain length was kept constant, and the number of chains in the system was increased up to a point in which the surface is saturated, i.e., unable to adsorb an additional chain. The systems will be denoted, respectively, as set 1 and set 2 .

The parameters varied in the work together with a brief description of the labels employed in Table I are summarized. A surface of constant size, comprising 100 charge points regularly distributed and spaced by $16 \AA$ along the $x$ and $y$ directions in a square grid, was considered. The surface size was chosen to be large enough to allow a complete adsorption of the longest chain considered. In Figure 1 some snapshots of the systems under study are depicted. Note, in panel (a), an illustration of the complete adsorption of the longest chain considered.

Note also that, as the surface size is smaller than the chain dimension, some compaction occurs in order to maximize the interaction between the chain and the surface. From panel (b) to (f), chains are progressively smaller and the adsorption to the surface is, a priori, possible without significantly affect the respective bulk conformation. The progressive surface coverage with chains of constant length is depicted in panels (g)-(l). It can be seen that, as the number of chains increases, the growing repulsion induces some degree of desorption, which becomes particularly evident in panels $(\mathrm{k})$ and (1).

For each system considered, the extent of adsorption, the corresponding degree of surface charge compensation, 
(a)

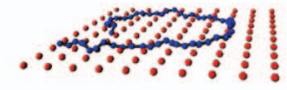

(b)

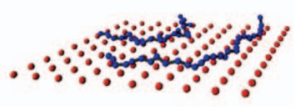

(c)

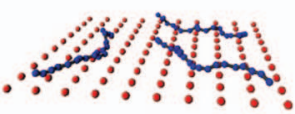

(d)

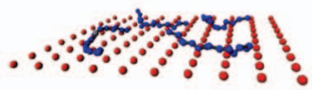

(e)

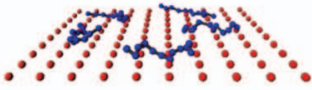

(f)

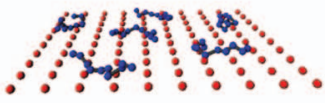

(g)

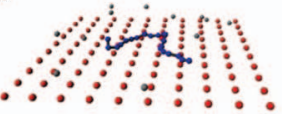

(h)

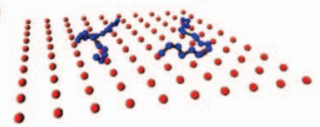

(i)
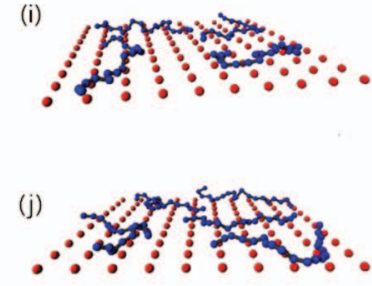

(k)

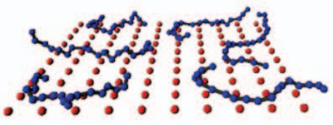

(1)

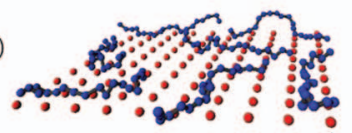

FIG. 1. Representative snapshots of negatively charged polyelectrolyte chains (blue) adsorbing onto a positive regularly charged surface (red) for different number of chains and chain lengths. Surface and chain counterions are represented in grey. All particles have hard-sphere radius of $2 \AA$. (a) system C1n60; (b) system C2n30; (c) system C3n20; (d) system C4n15; (e) system C5n12; (f) system C6n10; (g) system C1n20; (h) system C2n20; (i) system C4n20; (j) system C5n20; (k) system C6n20; (1) system C7n20.

and the conformation of the polyelectrolyte on the surface was inspected. Additionally, the spatial organization of the polyelectrolyte chains on the surface was evaluated resorting to positioning maps and to the described proximity-based approaches.

\section{A. Does the number of chains control adsorption?}

The adsorption degree, considered as the ratio between the number of adsorbed segments and the total number of chain segments, is represented in Figure 2, as a function of the number of chains in the system. It can be seen that, in both sets, adsorption slightly decreases as the number of chains increases. It can also be seen that up to five chains, the degree of adsorption is very similar in both sets, suggesting a significant dependence on the number of chains. Other factors, such as chain length, play only a minor role in the range under study. This seems to be associated to some entropic factor, related with chain ends and respective mobility. For more than five chains, there is a marked decrease in adsorption for set 2 , reflecting surface saturation and subsequent charge reversal. Figure 3 represents the percentage of surface charge compensation, determined considering the contribution of all species in the systems, again, as a function of the number of adsorbed

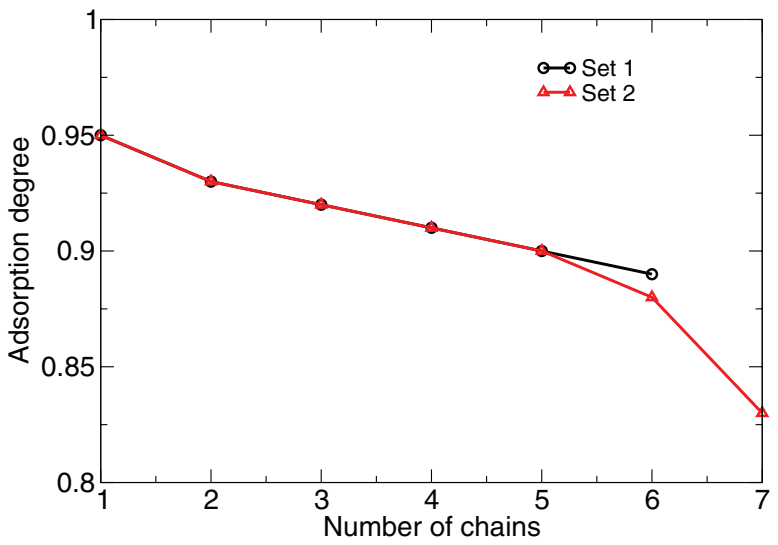

FIG. 2. Adsorption degree for polyelectrolyte chains of different (set 1) or constant (set 2) chain lengths, as a function of the number of chains in the system.

chains. It should be stressed that the contribution of the counterions to the surface charge compensation is always very small. Chain counterions, which act in the opposite way of surface charge compensation, contribute less than $0.3 \%$, while surface counterions have a contribution to the surface charge compensation below $1 \%$ in systems of set 1 , and range from $\sim 8 \%$ to $0.3 \%$ in systems $\mathrm{C} 1 \mathrm{n} 20$ to $\mathrm{C} 7 \mathrm{n} 20$ (data not shown). Panel (a) reflects the proportionality between the degree of adsorption and the surface charge compensation and shows that the latter tends to decrease when smaller fragments are considered. In contrast, for set 2, panel (b), the charge compensation increases monotonically with the number of chains, attaining a certain degree of charge reversal which is maximal for seven chains. It should be noted that the surface is not able to adsorb an eighth chain.

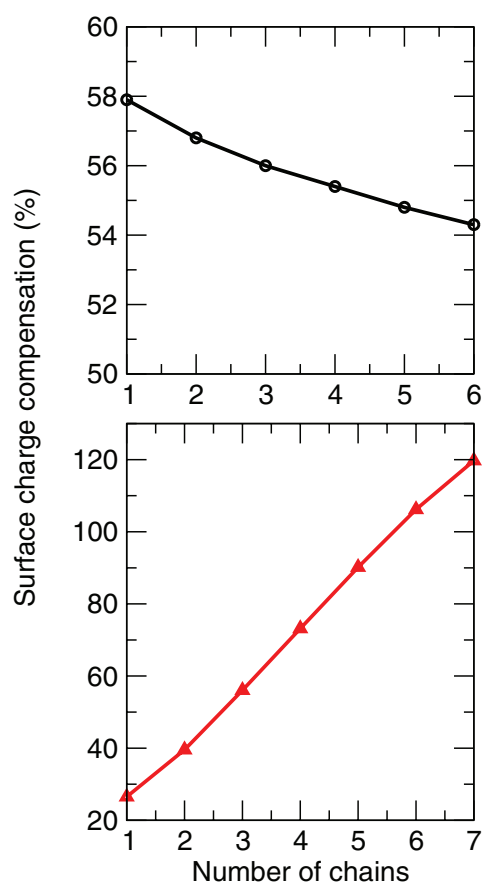

(a)

(b)

FIG. 3. Surface charge compensation as a function of the number of chains, in systems of set 1, panel (a), and set 2, panel (b). 


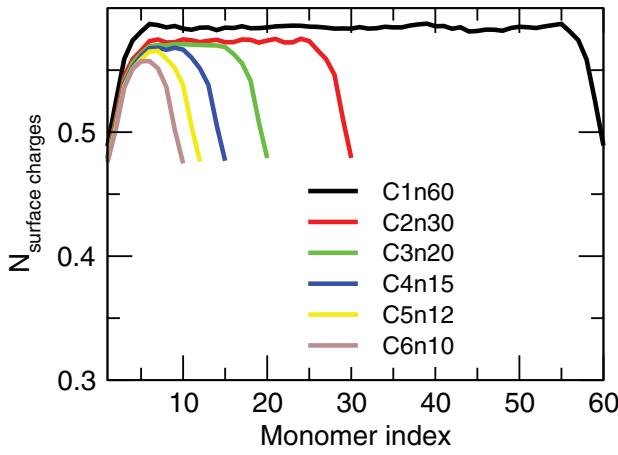

(a)

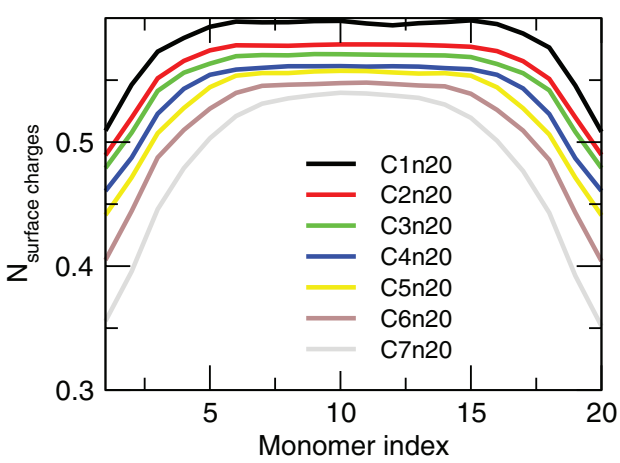

(b)

FIG. 4. Average number of surface charges at a distance lower than $8 \AA$ from a chain monomer, as a function of the monomer index, for systems of set 1 (a) and set 2 (b).

The profiles of the contact of chain monomers with the surface charges were also inspected, and are presented in Figure 4. The curves represent the average number of surface charges in contact with a chain monomer. These were calculated from the probability of finding a chain segment at a distance below $8 \AA$ from any surface charge. It can be seen that, independently of the system considered, the contact with the surface charges is considerably lower for the end monomers. For the shorter chains of set 1 , a decrease in contact is visible immediately after the central monomers. Also, there is a decrease in the overall contact as the number of chains increases, for both sets 1 and 2 . This decrease is more pronounced in the latter, due to the increased surface charge compensation and interchain repulsion.

It is also noteworthy that independently of the number and length of the chains, the chain contact occurs, on average, with less than 0.6 surface charges. Note that this contact analysis is more stringent than the one corresponding to monomer-wise adsorption, but the overall indications are compatible.

\section{B. Adsorption does not promote strong compaction}

In order to characterize the conformation of the chains upon adsorption, the radius of gyration, $R_{g}$, was used. Due to the variation of the chain length imposed in set 1 , the values are reported relatively to those of the bulk, as indicated in Figure 5. In each case, the bulk value of the radius of gyration was obtained considering a single chain in a bulk phase.

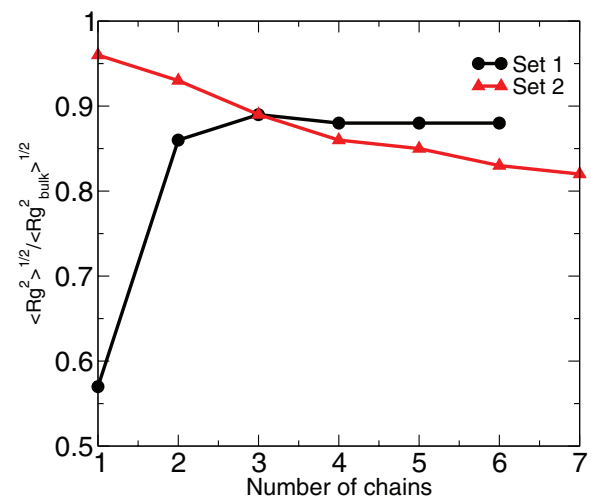

FIG. 5. Compaction degree as a function of the number of chains, evaluated by $R_{g}$ values relatively to the bulk, for systems of sets 1 and 2 .

In set $1, R_{g}$ increases as the fragmentation of the chain occurs. The adsorption of the longer chain $(\mathrm{C} 1 \mathrm{n} 60)$ imposes some degree of compaction, which becomes progressively lower as the chain length decreases, in spite of the increase in the number of chains. In set 2 , the reverse situation occurs. As the number of chain monomers is constant, a higher number of chains promote a higher degree of surface coverage, and some compaction occurs. Note, however, that this is a minor effect, in spite of the repulsion between chains. Apparently, the combined effect of adsorption and interchain repulsion does not significantly perturb the bulk conformation of the chains, which seems to be governed essentially by intrachain effects.

The extension of the polyelectrolyte chains normal and parallel to the surface plane was also quantified using the components of the radius of gyration perpendicular $\left(R g_{z}\right)$ and parallel to the surface plane $\left(R g_{x y}\right)$. Panel (a) of Figure 6 shows that $R g_{x y}$ decreases as the number of chains increases, both in sets 1 and 2. Naturally, this trend was expected in set 1, since the increase in the number of chains implies a decrease in their length. In set 2, the decrease of $R g_{x y}$ is dictated by the lowering of adsorption as the number of chains in the system grows. The $R g_{z}$ component, represented in panel (b) of Figure 6 shows an increasing trend for both sets 1 and 2, clearly more marked in the latter. In the former, a slight increase is found from one to two chains but the value is almost constant from there on. This trend is again related to the decrease in adsorption.

\section{Chain deposition favors specific regions of the surface}

The arrangement of the chains on the surface was inspected resorting to positioning density maps. In these representations, the probability of finding a monomer of the chain adsorbed at each point of the surface is plotted, relative to that found for a uniform distribution. The results are summarized in Figures 7 and 8, respectively, for systems of sets 1 and 2. In the same figures selected examples of some of the most frequent configurations found in each case are also included.

Figure 7 shows that when only one, relatively long chain is present, adsorption occurs preferentially in a ring pattern, 


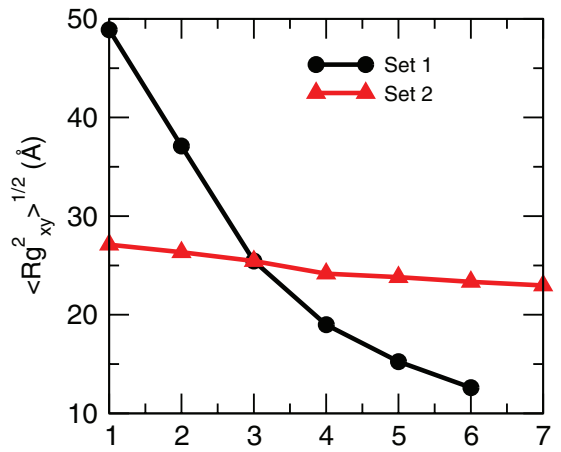

(a)

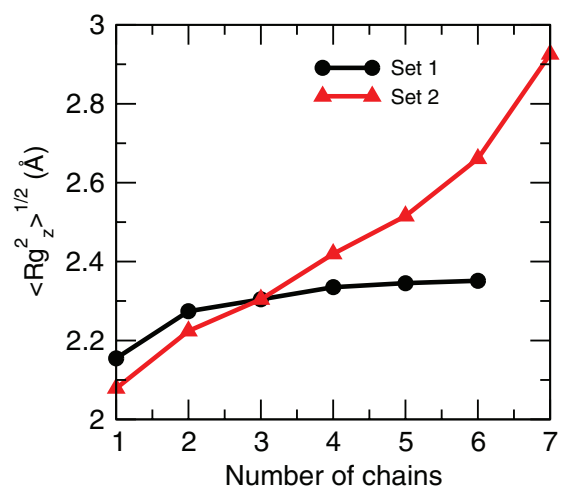

(b)

FIG. 6. Component of the radius of gyration of the polyelectrolyte chains, as a function of the number of chains, for systems of sets 1 and 2 in the $x y$ plane (a) and in the $z$-direction (b)

with the chain avoiding simultaneously the center and the periphery of the surface. This pattern can be understood considering the attractive potential of the surface, previously determined. ${ }^{40}$ It was found that, although the surface is composed of a uniform distribution of charges, the attractive potential created is non uniform. It is higher at the center of the surface and diminishes towards the periphery. When only one chain is present, under the influence of this attractive potential, the polyelectrolyte, ensuring a minimal bending penalty, seeks the region of the surface that maximizes the interaction. ${ }^{40}$ Naturally, when more than one chain is present, the effect of repulsion between chains must also be accounted for. The backbones occupy preferentially the regions of the surface where the attractive potential is higher, mainly avoiding the periphery of the surface, but interchain repulsion seems to dominate the distribution in this more favorable region, keeping the chains apart. Consequently, when two chains are present they are preferentially parallel to each other. When three chains are present, an almost equilateral triangular arrangement of the central monomers is observed (see Figure 7, system C3n20). It should be stressed that all the points around the center of the surface are equally probable, thus leading to an isodiametric "circular shape" 49 similar to the simplest and best known Releaux polygon. ${ }^{49-54}$ When the number of chains increases to four, the arrangement occurs preferentially at the corners of a square around the central part of the surface where the attractive potential is higher. Again, the chains avoid the periphery of the surface. For five and six chains, the pattern is more diffuse, but the chains tend to stay as distant as possible. The central part of the surface becomes now increasingly occupied.

In Figure 8 the same analyses are depicted for set 2 . There is a strong similarity between the behavior in the two sets. However, some differences occur because the chain lengths are not the same in the two sets. Therefore, in system C1n20, the polyelectrolyte occupies preferentially the central part of the surface, where the attractive potential is higher. The chain is relatively short and can be accommodated in this region of the surface, without a significant bending. For systems C2n20 to $\mathrm{C} 7 \mathrm{n} 20$ the arrangement is essentially the same observed in Figure 7. For a number of chains above three, the longer chains induce a higher uniformity on surface coverage, promoting the occupancy of the peripheral region of the surface, which was largely vacant for the smaller chain lengths of set 1 . Again, the chains tend to maintain a minimal compaction and be aligned, maximizing the relative distances.

\section{Chain ends play a major role in chain positioning}

After the characterization of general aspects of the adsorption process, focus is now given to the metrics pertaining to the relative arrangement of the chains. To this purpose, the nearest neighbor distances between chains and their relative orientation were first assessed. In Figure 9 the frequencies of the nearest neighbor distance between the central monomers of the chains are represented.

It is seen that, in set 1 , panel (a) when the number of chains increases, the distance between nearest neighbors tend to decrease. In set 2 , this monotonic trend is altered. While in the sequence three to seven chains the distances decrease, because of surface crowding, for two chains, distances fall between those found for C4n20 and C6n20, and strongly overlap with those of C5n20. This agrees with the pattern displayed in Figure 8, which suggests that the two chains are mainly close to the center of the surface, aligned and in a rod-like conformation.

In another, related, perspective, Figure 10 represents the distribution of the average nearest neighbor distances relatively to each monomer of the chain. In panel (a), set 1 , the profile is nearly independent of the number of chains. Note, however, the slight difference in the case of two chains, where there is a very slight minimum located close to each end, imputable to some bending of the end part of the chain. For the remaining systems, the end monomers are always the closest segment to the nearest chain, while the central part is the furthest apart. As the number of chains increases they become closer to each other, in spite of being increasingly shorter. This is a direct result of the higher potential in the center of the surface that promotes a certain degree of confinement: as the chains become shorter the repulsion decreases, which allows a closer proximity. In set 2, panel (b) the profile is more dependent on the number of chains. When two chains are present, the distribution is nearly uniform. It can be inferred that, in this case, the chains are considerably more spaced than in the systems containing a higher number of chains. This seems to contradict the results from Figure 9. However, the latter has to do with central monomer distances, while Figure 10 

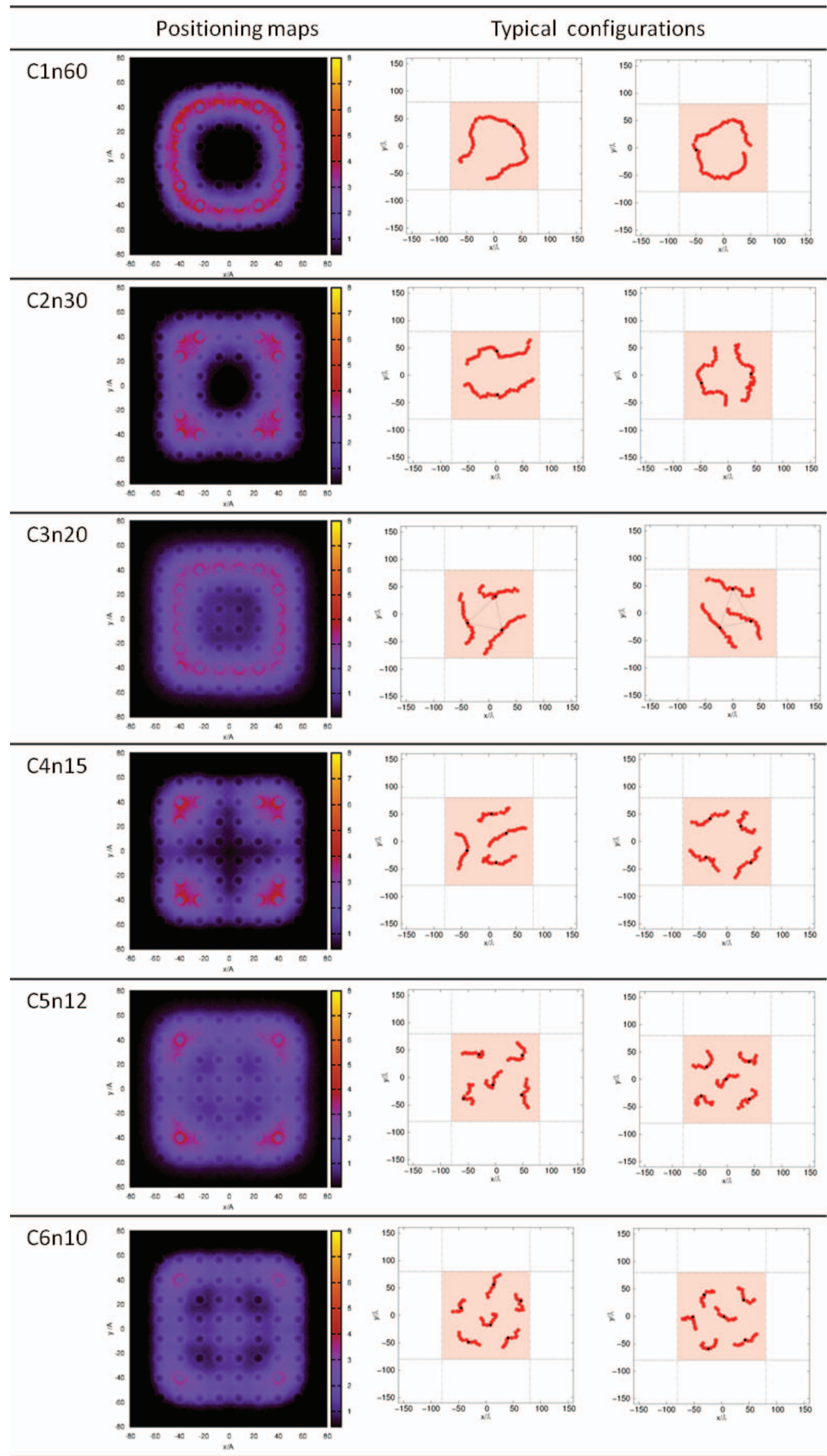

FIG. 7. Positioning maps for systems of set 1 , together with two representative configurations, for each system. The shaded regions in the two columns at right represent the surface area. 

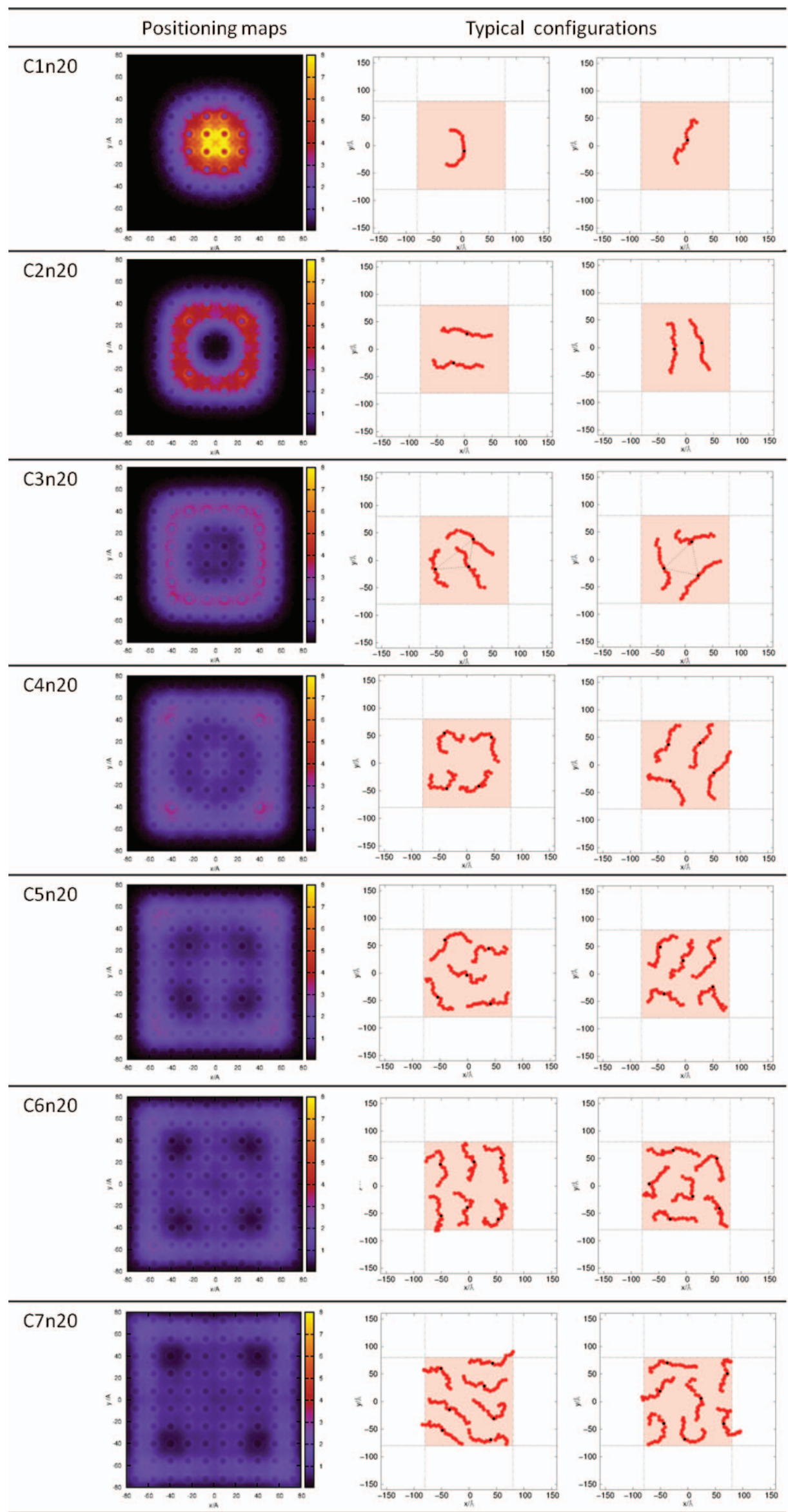

FIG. 8. Positioning maps for systems of set 2, together with two representative configurations, of each system. The shaded regions in the two columns at right represent the surface area. 


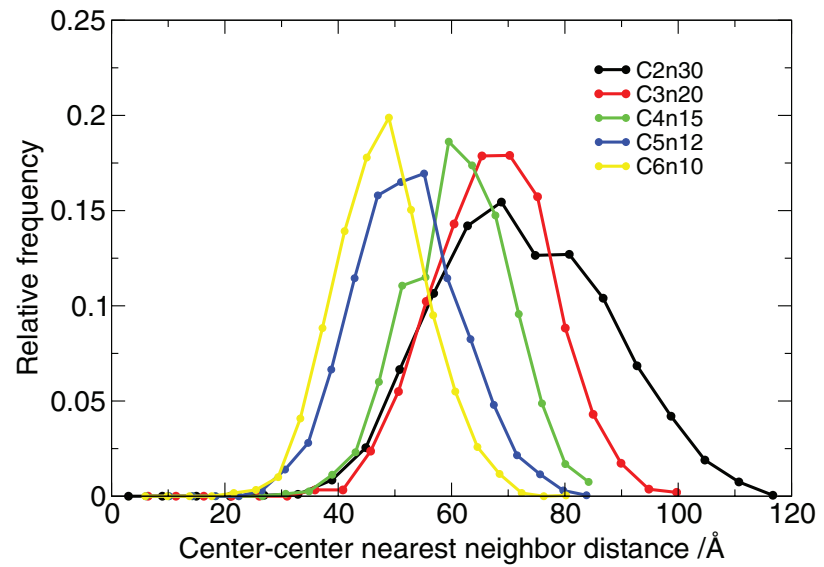

(a)

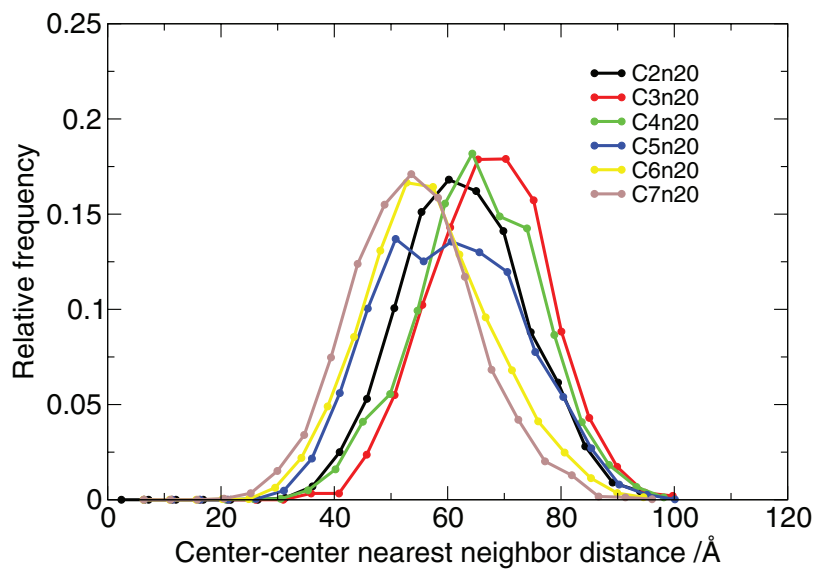

(b)

FIG. 9. Frequency of each nearest neighbor distance found between the central monomers of the chains. Panel (a) refers to systems of set 1 and panel (b) to those of set 2 .

deals with monomer to monomer proximity. As the number of chains increases, they tend to be closer to each other and, clearly, the end monomers are those closer to the neighboring chains. As in set 1 , the increasing number of chains induces a higher approximation between them, with the corresponding decrease in the nearest neighbor distances. In set 2 , this is motivated by the longer chain that naturally impose a higher approximation despite the increased repulsion. The flattening observed in the central part of some of the curves in panel (b) of Figure 10 suggests that, in those systems, chains are disposed in a parallel arrangement.

In Figure 11 the proximity profile between chains for set 1 is depicted. The profiles were obtained identifying the monomer responsible for the closest approximation between neighboring chains. These monomers are then counted and accumulated considering the respective index, and the result plotted in the form of the respective relative frequency. In panel (a) of Figure 11 corresponding to set 1, it can be seen that generally, and independently of the number of chains in the system, ends are closer to ends. Panel (b) of the same figure shows that, surprisingly, chain ends are also the monomers more often found closer to central monomers, and only in system $\mathrm{C} 2 \mathrm{n} 30$ this trend is somewhat altered. In this system cen-

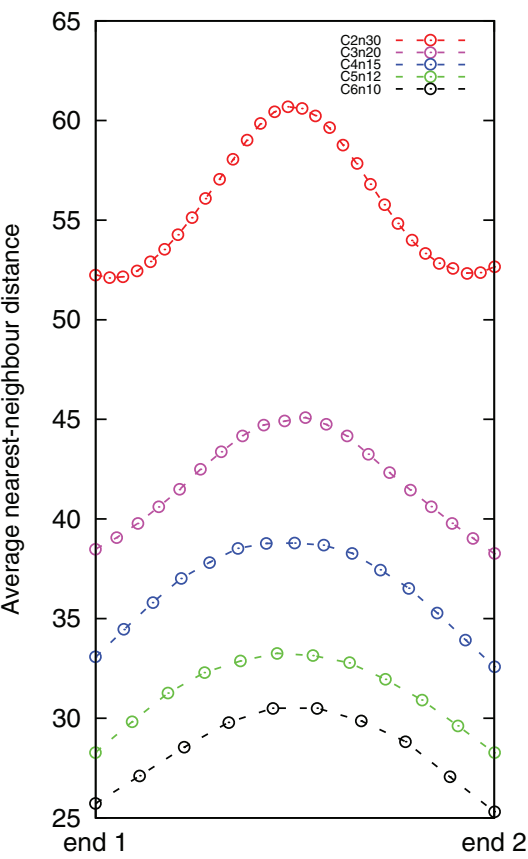

(a)

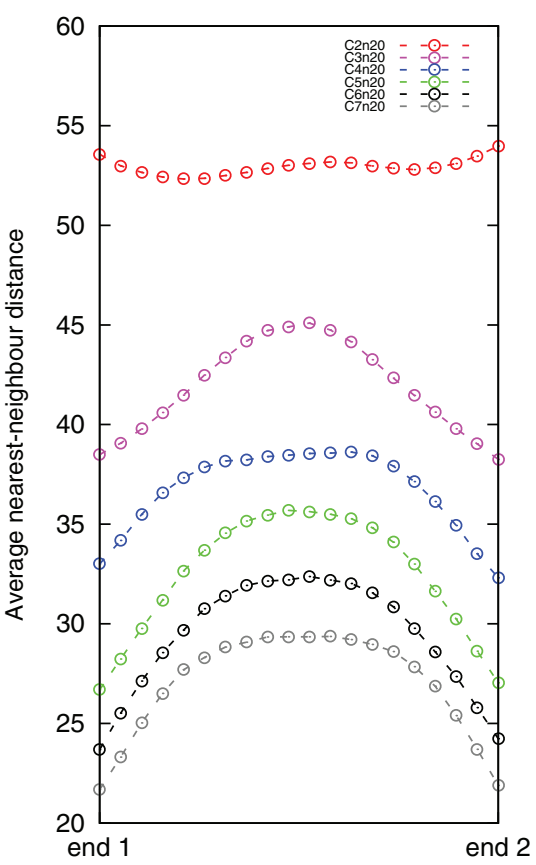

(b)

FIG. 10. Average nearest neighbor distance between each monomer of the chain and the neighboring monomer in another chain. Panel (a) refers to set 1 and panel (b) to set 2 .

tral monomers are also frequently found close to other central monomers. Very similar trends are observed for set 2 (data not shown).

The preference for chain ends as the site of closest proximity between chains can be understood inspecting the potential created by each chain, the whole set of chains and, also, the surface charges. While surface charges are attractive centers, chains repeal each other. In Figure 12 the electrostatic potential created by one and three chains in a typical configuration for system $\mathrm{C} 3 \mathrm{n} 20$ is represented, the latter with and 


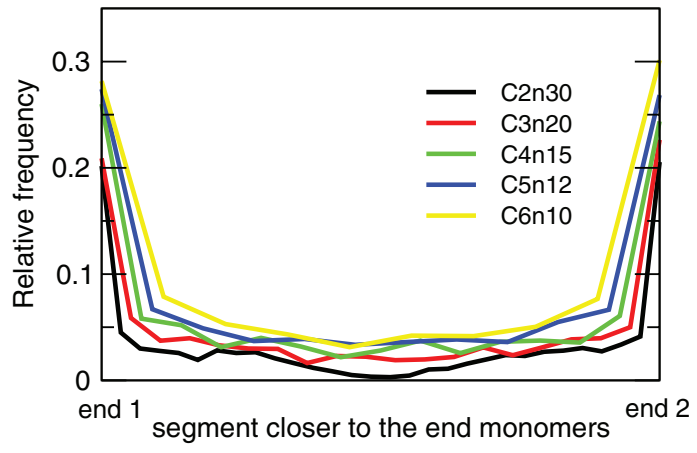

(a)

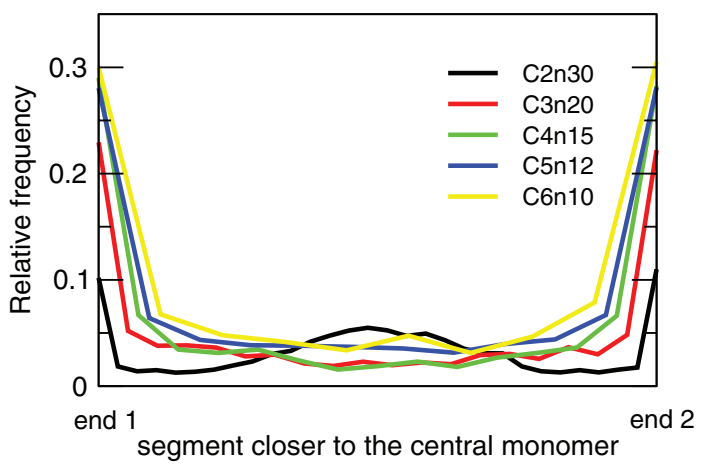

(b)

FIG. 11. Relative frequency of the monomers (represented by the respective index) closest to the end (a) or central (b) monomers of the neighboring chain, for systems of set 1 .

without the attractive contribution of the surface charges. In panels (a) and (b), in which the effect of surface charges is not included, it is clear that the vicinity of the chain ends is less repulsive than the middle part. It is noteworthy that the repulsive potential created by the three chains is magnified in the region between them, due to their cumulative contribution. However, and as stated before, the potential created by the surface charges is higher in the surface center, and under the influence of this attractive potential, chains tend to approach the center. This effect promotes a more homogeneous potential in the surface (Figure 12(c)).

After discussing the rationale for the monomers involved in the closest proximity with neighboring chains, the average distance between chain ends, ends-central monomers, and between central monomers, the latter only in systems C2n20 and $\mathrm{C} 2 \mathrm{n} 30$, is presented in Table II. Results indicate that the distance of closest approach between chains does not significantly depend on the sites involved, which is compatible with the relatively uniform potential created. Naturally, these distances decrease as the number of chains increases. Set 2 behaves in a similar fashion.

\section{E. Overview}

In summary, it was found that independently of the polyelectrolyte chain length and degree of surface charge neutralization, the contact of chain ends with surface charges is always weaker than that of the remaining backbone. This occurs

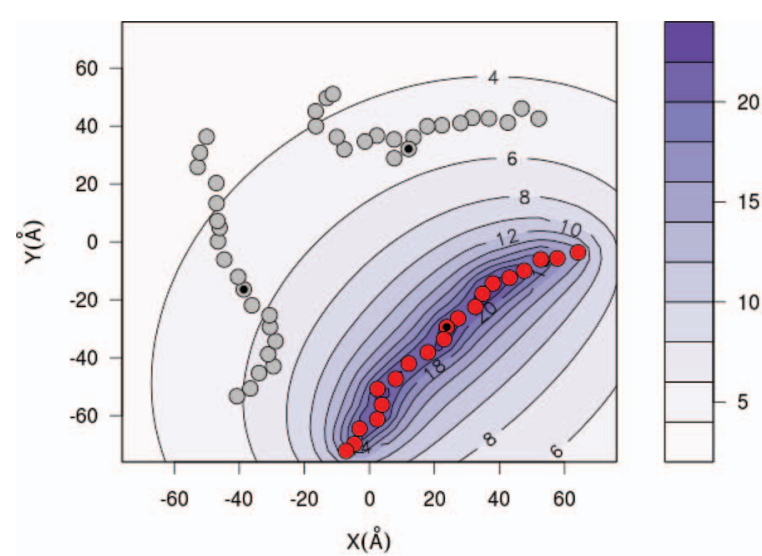

(a)

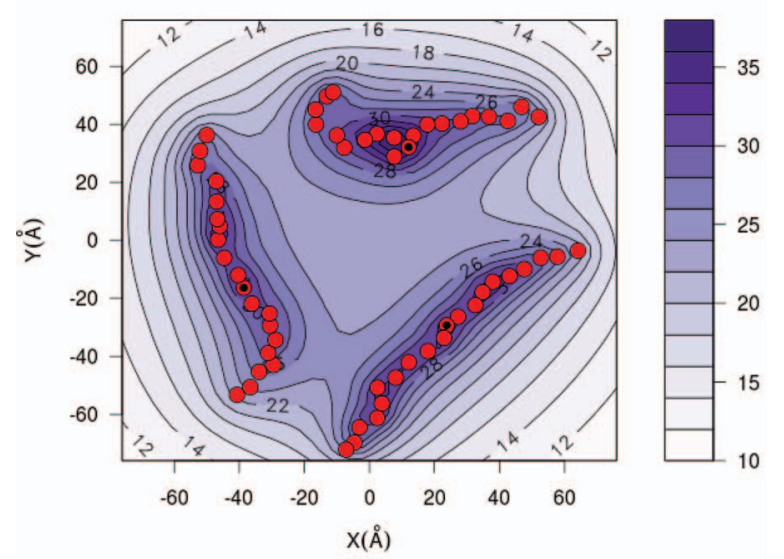

(b)

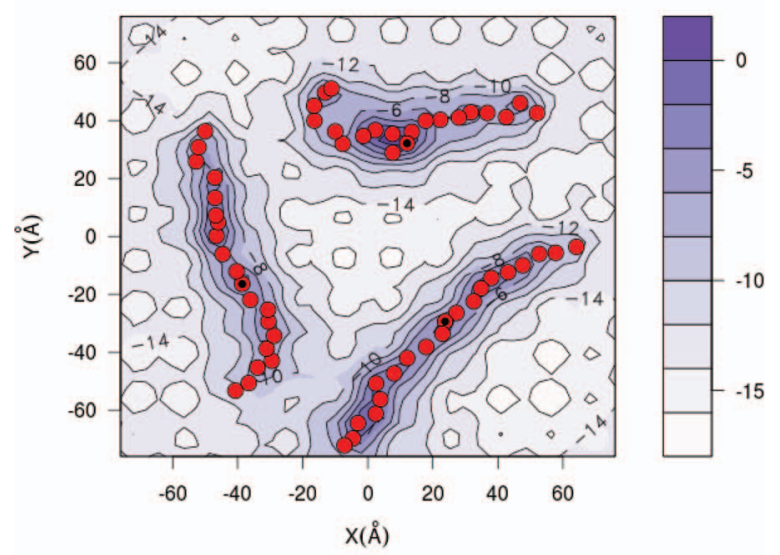

(c)

FIG. 12. Potential energy contours of the Coulombic energy (kJ/mol) calculated considering the effect of only one (a) and three chains (b), without contribution of the surface charges, in a typical configuration for system $\mathrm{C} 3 \mathrm{n} 20$. In panel (c), the attractive contribution of the surface charges was also included.

even for very small chains, and in situations where the surface is markedly undercharged.

It was also seen that adsorption occurs mainly in flat arrangements parallel to the surface, as already observed in other systems, ${ }^{27,55,56}$ even in electrically neutral surfaces. ${ }^{56}$ Moreover, this occurs without a significant compaction of the chains. The interaction between polyelectrolyte and surface promotes a spreading of the backbones dictated by electrostatics. This is valid even when surface overcharging occurs, 
TABLE II. Values of the average nearest neighbor distance \pm SD between the indicated closest monomers of neighboring chains.

\begin{tabular}{lccc}
\hline \hline System & & Distance $(\AA)$ & \\
\hline & End-end & End-central & Central-central \\
Set 1 & & & \\
C2n30 & $46 \pm 18$ & $43 \pm 17$ & $45 \pm 17$ \\
C3n20 & $41 \pm 15$ & $43 \pm 16$ & $\ldots$ \\
C4n15 & $42 \pm 15$ & $41 \pm 15$ & $\ldots$ \\
C5n12 & $38 \pm 14$ & $37 \pm 14$ & $\ldots$ \\
C6n10 & $36 \pm 12$ & $36 \pm 13$ & $\ldots$ \\
Set 2 & & & \\
C2n20 & $50 \pm 15$ & $50 \pm 16$ & $\ldots 6 \pm 13$ \\
C3n20 & $42 \pm 16$ & $43 \pm 16$ & $\ldots$ \\
C4n20 & $40 \pm 15$ & $40 \pm 15$ & $\ldots$ \\
C5n20 & $35 \pm 13$ & $35 \pm 14$ & $\ldots$ \\
C6n20 & $32 \pm 13$ & $33 \pm 13$ & $\ldots$ \\
C7n20 & $31 \pm 14$ & $31 \pm 13$ & $\ldots$ \\
\hline \hline
\end{tabular}

and is reflected on the $R_{g}$ values, that do not change significantly relatively to the bulk phase. Apparently, the balance between adsorption and interchain repulsion does not considerably affect the bulk conformation, indicating that the repulsive interactions between the charged segments dominate over interchain repulsion. This is in accordance with the behavior previously reported ${ }^{57}$ in a study on the adsorption of amphiphilic polyelectrolytes on silica, at low salt concentration. It also agrees with observations on the adsorption of uncharged homopolymers to surfaces, ${ }^{58}$ in which flexible chains retain most of the respective conformational freedom upon adsorption.

It was also found that arrangements in which the ends are the sites of closest proximity between chains are dominant. Ends are also the prevalent sites of closest proximity to central monomers. The lower potential created at the chain ends, makes this part of the backbone the less adsorbed one, and induces a frequent end-pointing-to-end relative positioning, in order to minimize interchain repulsion.

In what regards the patterns of adsorption, results indicate that chains are located preferentially in the regions of the surface where the attractive potential is higher, and the periphery becomes occupied only when surface overcharge is attained. Furthermore, interchain repulsion dictates the distribution of the chains on the favorable region of the surface. Irrespective of the arrangement of the chains, the shortest distance between them is approximately constant and independent of the monomers responsible for this closest proximity. As the number of chains increases, this distance tends to decrease, again, irrespective of the monomer pair involved.

\section{CONCLUSIONS}

Two sets of systems comprising an increasing number of chains of different or constant chain length adsorbing on a oppositely charged surface with a regular charge distribution were examined.

The aim of the work was to improve the understanding of the structure of the adsorbed layer, resorting to a very simple model, and thus give a contribute to areas involving polyelec- trolyte deposition. To achieve this goal, the factors that control the conformation and the patterns of adsorption of multichain like-polyelectrolytes were inspected in detail.

Considering the nature of the systems under study, and the surface-polyelectrolyte affinity, a regular adsorption could be expected. Instead, a feature rich behavior was observed.

\section{ACKNOWLEDGMENTS}

This work was supported by FEDER funds through the COMPETE program-Programa Operacional Factores de Competitividade - and by National funds through Fundação para a Ciência e Tecnologia(FCT) under the Project PTDC/QUI-QUI/101442/2008 (COMPETE: FCOMP01-0124-FEDER-010831). T.F.G.G.C. acknowledges the same project for a B.I. research grant. S.C.C.N. gratefully acknowledges the post-doctoral research Grant No. SFRH/BPD/71683/2010 assigned by the Fundação para a Ciência e Tecnologia (FCT).

${ }^{1}$ A. S. Malinin, I. V. Kalashnikova, A. A. Rakhnyanskaya, and A. A. Yaroslavov, Polym. Sci., Ser. A 54, 81-86 (2012).

${ }^{2}$ D. L. Elbert, C. B. Herbert, and J. A. Hubbell, Langmuir 15, 5355-5362 (1999).

${ }^{3}$ M. Dimitrova, Y. Arntz, P. Lavalle, F. Meyer, M. Wolf, C. Schuster, Y. Haïkel, J.-C. Voegel, and J. Ogier, Adv. Funct. Mater. 17, 233-245 (2007).

${ }^{4}$ N. Benkirane-Jessel, P. Schwinte, P. Falvey, R. Darcy, Y. Haikel, P. Schaaf, J. C. Voegel, and J. Ogier, Adv. Funct. Mater. 14, 174-182 (2004).

${ }^{5}$ F. Caruso, K. Niikura, D. N. Furlong, and Y. Okahata, Langmuir 13, 34223426 (1997).

${ }^{6}$ F. Caruso, K. Niikura, D. N. Furlong, and Y. Okahata, Langmuir 13, $3427-$ 3433 (1997).

${ }^{7}$ F. Caruso, E. Rodda, D. F. Furlong, K. Niikura, and Y. Okahata, Anal. Chem. 69, 2043-2049 (1997).

${ }^{8}$ Y. Wang, L. Hosta-Rigau, H. Lomas, and F. Caruso, Phys. Chem. Chem. Phys. 13, 4782-4801 (2011).

${ }^{9}$ M. I. Toral, J. González-Navarrete, A. Leiva, H. E. Ríos, and M. D. Urzúa, Eur. Polym. J. 45, 730-737 (2009).

${ }^{10}$ L. Wagberg, L. Winter, L. Odberg, and T. Lindstrom, Colloids Surf. 27, 163-173 (1987).

${ }^{11}$ D. Volodkin, Y. Arntz, P. Schaaf, H. Moehwald, J.-C. Voegel, and V. Ball, Soft Matter 4, 122-130 (2008).

${ }^{12}$ H. Kerdjoudj, N. Berthelemy, F. Boulmedais, J.-F. Stoltz, P. Menu, and J. C. Voegel, Soft Matter 6, 3722-3734 (2010).

${ }^{13}$ F. Zhou and W. T. S. Huck, Phys. Chem. Chem. Phys. 8, 3815-3823 (2006).

${ }^{14}$ S. M. Notley, J. Colloid Interface Sci. 375, 35-40 (2012).

${ }^{15}$ G. Decher, Science 277, 1232-1237 (1997).

${ }^{16}$ Y.-C. Chang and C. W. Frank, Langmuir 12, 5824-5829 (1996).

${ }^{17}$ Y. Wang, Q. Hong, Y. Chen, X. Lian, and Y. Xiong, Colloids Surf., B 100, 77-83 (2012).

${ }^{18}$ A. G. Cherstvy and R. G. Winkler, Phys. Chem. Chem. Phys. 13, 1168611693 (2011).

${ }^{19}$ M. Manghi and M. Aubouy, Phys. Chem. Chem. Phys. 10, 1697-1706 (2008).

${ }^{20}$ E. Seyrek, J. Hierrezuelo, A. Sadeghpour, I. Szilagyi, and M. Borkovec, Phys. Chem. Chem. Phys. 13, 12716-12719 (2011).

${ }^{21}$ A. G. Cherstvy and R. G. Winkler, J. Phys. Chem. B 116, 9838-9845 (2012).

${ }^{22}$ R. Messina, Macromolecules 37, 621-629 (2004).

${ }^{23}$ C. F. Narambuena, D. M. Beltramo, and E. P. M. Leiva, Macromolecules 40, 7336-7342 (2007)

${ }^{24}$ J.-M. Y. Carrillo and A. V. Dobrynin, Langmuir 23, 2472-2482 (2007).

${ }^{25}$ C. Y. Kong and M. Muthukumar, J. Chem. Phys. 109, 1522-1527 (1998).

${ }^{26} \mathrm{Q}$. Wang, Soft Matter 5, 413-424 (2009).

${ }^{27}$ B. Qiao, J. J. Cerdà, and C. Holm, Macromolecules 44, 1707-1718 (2011).

${ }^{28}$ R. R. Netz and J.-F. Joanny, Macromolecules 32, 9013-9025 (1999).

${ }^{29}$ M. Schönhoff, J. Phys.: Condens. Matter 15, R1781-R1808 (2003).

${ }^{30}$ P. A. Patel, J. Jeon, P. T. Mather, and A. V. Dobrynin, Langmuir 22, 9994 10002 (2006). 
${ }^{31}$ B. Qiao, M. Sega, and C. Holm, Phys. Chem. Chem. Phys. 13, 1633616342 (2011).

${ }^{32}$ C. F. Narambuena, D. M. Beltramo, and E. P. M. Leiva, Macromolecules 41, 8267-8274 (2008).

${ }^{33}$ R. Messina, Phys. Rev. E 70, 051802 (2004).

${ }^{34}$ C. N. Patra, R. Chang, and A. Yethiraj, J. Phys. Chem. B 108, 9126-9132 (2004).

${ }^{35}$ R. S. Dias, A. A. C. C. Pais, P. Linse, M. G. Miguel, and B. Lindman, J. Phys. Chem. B 109, 11781-11788 (2005).

${ }^{36}$ M. Ellis, C. Y. Kong, and M. Muthukumar, J. Chem. Phys. 112, 8723-8729 (2000).

${ }^{37}$ W. Fang, Y. Cai, X. Chen, R. Su, T. Chen, N. Xia, L. Li, Q. Yang, J. Han, and S. Han, Bioorg. Med. Chem. Lett. 19, 1903-1907 (2009).

${ }^{38}$ M. Urzúa, X. Briones, L. P. Carrasco, M. Encinas, and D. Petri, Polymer 51, 3445-3452 (2010).

${ }^{39}$ X. G. Briones, M. V. Encinas, D. F. S. Petri, J. E. Pavez, R. A. T. M. Yazdani-Pedram, and M. D. Urzúa, Langmuir 27, 13524-13532 (2011).

${ }^{40}$ S. C. C. Nunes, P. Pinto, and A. A. C. C. Pais, J. Comput. Chem. 34, 1198 1209 (2013).

${ }^{41}$ R. Messina, J. Chem. Phys. 124, 014705 (2006).

${ }^{42}$ O. V. Borisov, E. B. Zhulina, and T. M. Birshtein, J. Phys. II 4, 913-929 (1994).

${ }^{43}$ P. Linse, MOLSIM, version 4.0.8, 2004.

${ }^{44}$ M. P. Allen and D. J. Tildesley, Computer Simulation of Liquids (Clarendon, Oxford, 1987).
${ }^{45}$ M. Skepö, J. Chem. Phys. 129, 185101 (2008)

${ }^{46}$ A. Tulpar and W. A. Ducker, J. Phys. Chem. B 108, 1667-1676 (2004).

${ }^{47}$ O. J. Rojas, Encyclopedia of Surface and Colloid Science (Marcel Dekker, 2002), pp. 517-535.

${ }^{48}$ J. W. Eaton, D. Bateman, and S. Hauberg, GNU Octave Manual: A Highlevel Interactive Language for Numerical Computations (Network Theory Ltd., 2007).

${ }^{49}$ J. Buescu, Ingenium Series II, 129, 110-111 (2012) (in Portuguese).

${ }^{50}$ J. Brunhorn, O. Tenchio, and R. Rojas, RoboCup 2006: Robot Soccer World Cup X, Lecture Notes in Artificial Intelligence Vol. 4434 (SpringerVerlag, Berlin, 2007), pp. 516-522.

${ }^{51}$ Y. Miao, G. Yan, and Z. Lin, Int. J. Innov. Comput. Inf. and Control 7, 5851-5864 (2011)

${ }^{52}$ M. J. Mossinghoff, J. Comb. Theory, Ser. A 118, 1801-1815 (2011).

${ }^{53}$ A. Webster, Mon. Not. R. Astron. Soc. 353, 1304-1310 (2004).

${ }^{54}$ E. Munoz-Sandoval, J. J. Torres-Heredia, and F. Lopez-Urias, J. Appl. Phys. 97, 10E318 (2005).

${ }^{55}$ H. Ahrens, H. Baltes, J. Schmitt, H. Möhwald, and C. A. Helm, Macromolecules 34, 4504-4512 (2001).

${ }^{56}$ A. A. Chialvo and J. M. Simonson, J. Phys. Chem. C 112, 19521-19529 (2008).

${ }^{57}$ Y. Samoshina, T. Nylander, P. Claesson, K. Schillén, I. Iliopoulos, and B. Lindman, Langmuir 21, 2855-2864 (2005).

${ }^{58}$ P. Linse and N. Källrot, Macromolecules 43, 2054-2068 (2010). 